\title{
EVALUATION OF COTTON AND POLYTETRAFLUOROETHYLENE TAPE AS ENDODONTIC SPACER MARTIAL IN PULPECTOMY OF PRIMARY MOLARS
}

\author{
Arafa Khatab* and Lina Jamil Abdelhafez**
}

\begin{abstract}
Aim: This study aimed to evaluate the sealing ability of cotton and Polytetrafluoroethylene (PTFE) tape as spacer under temporary filling material in between root canal treatment visits.

Method and Materials: forty infected primary molars indicated for pulpectomy from twenty children were selected for this study. Two visits root canal treatment were done with temporary restorative material and application of the tested cotton and PTFE as a spacers on each side randomly. Microbiological study was done after pulpectomy by taking sample from the coronal portion of the cavity at the folloing steps, directly after pulpectomy, seven days follow up for both the cavity and the spacer material itself.
\end{abstract}

Results: Cotton fibers group showed bacterial contamination for both the cavity and material itself while PTFE showed the least amount of bacterial growth over their samples. There was contamination of the majority of cotton group samples in contrary to minimal amount for PTFE samples showed bacterial contamination.

Conclusions: Using PTFE as a spacer material under provisional restoration showed very good results from microbial point of view and it may be used as cotton alternative in the future in the endodontic field

Key words: Endodontic spacer, PTFE tape, Primary teeth, Pulpectomy, Microbial leakage

\section{INTRODUCTION}

Primary teeth are considered the best space maintainers. Maintaining function, esthetics, arch length symmetry and integrity of primary dentition till normal exfoliation are the main targets in addition of preventing of harmful psychological effects of tooth loss. ${ }^{(1-3)}$
Pulpectomy is a pulp therapy technique recommended for the treatment of irreversible pulpitis or necrosis caused by dental trauma or caries, and is considered a conservative option compared with tooth extraction ${ }^{(4)}$.

Pulpectomy could be performed in single visit or in multiple visits, the indications of each varies.

\footnotetext{
* Lecturer of Pediatric Dentistry, Tanta University.

** Lecturer Microbiology Department, Faculty of Pharmacy, 6 October University.
} 
Whenever a chronic infection, abscess, sinus or fistula exists or the pulp is necrotic, multiple visit pulpectomy is indicated. The use of provisional material is mandatory when pulpectomy is performed in multiple visits so as to prevent infection of root canals between the appointments ${ }^{(5)}$.

Failure to apply of the proper and perfect temporary restorations during endodontic treatment is one of the main causative factors in postoperative pain. Accordingly, temporary materials must provide an adequate seal against ingress of bacteria, fluids and organic materials from the oral cavity to the obturated root canals, and so the sterility must be in its highest level in between the appointments until a final coronal restoration is placed following root-canal obturation ${ }^{(6,7)}$.

The effect of coronal leakage on the results of endodontic treatment has been widely accepted ${ }^{(8)}$. Bacterial infection is one of the main factors associated with root canal failure ${ }^{(9)}$. Therefore, the main aims of the endodontic treatment are removing microorganisms from the root canal using both chemical and mechanical debridement, and sealing of the root canal system from irritants ${ }^{(10)}$, which may induce periapical lesions ${ }^{(11,12)}$, thus all effort should be done to prevent infection of the pulp space in each step of endodontic treatment. Several studies have demonstrated that the coronal seal as important as the quality of root canal filling for periapical health ${ }^{(\mathbf{1 3}, 14)}$. Microorganisms may able to go through root canal filling ${ }^{(15)}$ and coronal leakage may happen within a few days ${ }^{(\mathbf{1 3})}$. So, temporary restorative materials are usually used during root canal therapy to seal the root canal between visits or until a permanent restoration is placed.

The two main factors affecting the microbial leakage from the oral cavity to the obturated canals are thickness of the temporary restoration and type of spacer material. ${ }^{(16,17)}$.

Regarding the thickness of the temporary restorative material, minimum depth required to prevent leakage in the access cavity is $3.5 \mathrm{~mm}{ }^{(16)}$. Parris et al demonstrated leakage of provisional restorative materials and subsequent bacterial contamination of cotton pellets after only one week ${ }^{(18)}$.

Spacers are the materials placed between the canal orifice and the temporary restoration in between endodontic treatment visits for maximum sterility, also prevents the temporary restoration material from obstructing the canal orifices and allows its easy removal ${ }^{(19)}$.

The ideal requirements for spacer material it should be inert, inorganic, readily available, inexpensive, easy to use, autoclavable, easily visible, and easy to place and remove. Further, the spacer should take up minimal volume on the cavity and support the overlying restoration ${ }^{(17)}$.

Cotton consider the most widely used spacer beneath temporary restorative materials ${ }^{(20)}$. Conversely, its use could introduce complications to the required seal, including the following drawbacks, it may affect the thickness of the temporary restoration and lead to increased bacterial outflow, cotton threads could be incorporated within the temporary restorative material and reach on external surface of the material, moreover during the removal of the fibers of the cotton pellet may adhere to the walls of access cavity and serve as a wick, finally it may affect the stability of the restoration by acting as a cushion, allowing displacement during masticatory force $^{(21)}$.

Many studied were done in the way to found better alternative and avoid the cotton drawbacks as a spacer material. Foam pellets and Polytetrafluoroethylene (PTFE) tape were the first tried materials as spacers after cotton ${ }^{(\mathbf{1 7}, \mathbf{2 2})}$.

The researcher found in the PTFE which is commonly used as plumber's tape all the criteria which could overcome all the drawbacks of cotton (17). first of all it is ribbon like inorganic material which means that there is no possibility 
of bacterial uptake by wicking It is also non fibrous reducing the chances of getting impregnated within the temporary restorative material. It is non spongy, thus allowing better support to the overlying temporary restorative material ${ }^{(\mathbf{1 7})}$.

In vivo study of (PTFE) as spacer in primary teeth done by Attiguppe et al 2018, they made comparison between cotton and PTFE when used clinically as spacer in endodontically treated primary molars. They recorded very good microbiological results of PTFE against cotton and recommending its use as an alternative to cotton in primary teeth pulpectomy $^{(17)}$.

In another study done by Aditya et al $2019^{(23)}$ three materials were microbiologically evaluated as a spacer material which were, cotton, cellulose sponge and polytetrafluoroethylene tape (PTFE). They tried the three materials alone or after immersed in antiseptic material. The results revealed good results of cotton when used with medicament while PTFE was the best spacer when used alone .

This study was performed to evaluate the use of PTFE tape as endodontic spacer compared to cotton fibers.

\section{MATERIALS AND METHODS}

This clinical trial study was carried out on twenty healthy children (with forty molar teeth indicated for pulpectomy) aged from six to eight years from patients attending outpatient clinic of Pediatric Dentistry Department Tanta University. The microbiological study was done at Laboratory of Microbiology, Faculty of Pharmacy, 6 October University.

\section{Ethical approval}

Written consents for treatment was obtained prior to the clinical procedures after the details of the treatment procedure were clear to the child's parents or guardians according to the guidelines on human research adopted by Ethics Committee, Faculty of Dentistry, Tanta University.

\section{Sample selection}

\section{Clinical criteria ${ }^{(17)}$}

The selected teeth had met one or more of the following criteria:

- Bilateral primary molars with necrotic pulp tissue upon entrance the pulp chamber.

- Presence of gingival swelling

- Spontaneous pain

- Pain on percussion.

\section{Radiographic criteria}

- Evidence periapical radiolucency

- Discontinuity of lamina dura

- Physiological root resorption not greater than one third of the root.

\section{Exclusion criteria}

The exclusion criteria were included the following:

- Not restorable tooth.

- Extensive root resorption more than one third of the root

- Pulpal floor perforation

- Patients selected for this study should be medically free from any chronic or infectious diseases as cardiac or diabetic patients.

A full-mouth examination for patients after complete history and personal data was carried out along with periapical radiographs for any teeth with possible indication of pulpectomy before the start of the clinical study. The forty selected primary molars were randomly divided by specialist pedodontist not involved in the study into two groups of twenty teeth for each, according to the spacer material used as follow: 
Group 1: Cotton as endodontic spacer (positive control group). $\bullet$

Group 2: Polytetrafluoroethylene tape as endodontic spacer (study Group) .

\section{Methods}

All treatments were carried out by the same operator. After confirming the diagnosis, local anesthesia was achieved, proper isolation of the teeth was done using rubber dam. All caries was removed with excavator and round tungsten carbide bur, deroofing of the pulp chamber was done with sterile diamond burs in a high-speed handpiece. The exposed inflamed coronal pulp tissue was amputated using a spoon excavator and irrigated with saline. Then, the necrosed tissue was carefully extirpated from the root canals using 15 size $\mathrm{H}$ file. The working length was determined by a radiograph. Root canal files were selected and adjusted to stop $1 \mathrm{~mm}$ short of the radiographic apex of each canal. After cleaning canals from pulp tissues, canals were debrided thoroughly, then mechanical preparation of the canals was done until size 30 . The canals were carefully irrigated with $1 \%$ sodium hypochlorite $(\mathrm{NaOCl})$ solution with no excessive pressure and finally with normal saline. The cleaned canals were dried using paper points and then obturated using Metapex (META BIOMED CO.LTD, Korea), Postoperative periapical $\mathrm{x}$ ray film was taken for confirmation of the accuracy of pulpectomy treatment and to check the accuracy of the obturation and there is optimal periapical seal (fig 1-6). The cavity after this was ready for the microbiological part of the study.

First sample was taken from the access cavity after complete cleaning and irrigation with normal saline by gently scrapping it with a sterile sharp spoon excavator (fig 2). The collected samples were placed into brain heart infusion broth. Sterilized Cotton pellets and PTFE tape ( PIAIN TEFLON BANT ,SINIF F,FRP:INCE VIDADISL
ERI, DN , ISO 9001:2000, 12 mm TURKY) of standardized size six centimeter) were then placed as spacers on each side (fig 3) according to study groups classification. Above the spacers Cavit G (3M ESPE, Germany) temporary restorative material of standardized thickness $(3.5 \mathrm{~mm})$ was placed. Patients were recalled after 7 days. (Fig 5).

After one week, provisional restoration was removed carefully by aseptic technique using micro motor (NSK, Japan) and using sterile tweezer. Spacer materials were transferred to brain heart infusion broth. After this, the samples were centrifuged at $2500 \mathrm{rpm}$ for 10 minutes in a centrifuge machine for homogenization of samples. A $0.1 \mathrm{ml}$ of sample was taken and inoculated on blood agar plates to check for microbial growth and bacterial colony count. Plates were incubated for $24-36$ hours at $37^{\circ} \mathrm{C}$ in an incubator. The plates were then examined and the number of bacterial colonies were counted using colony forming unit using a pen and click-counter in terms of $\mathrm{cfu} / \mathrm{ml}$ of the inoculum.

\section{Evaluation of the cavity contamination under- neath the tested materials}

After removal of the spacer materials and transferring it for microbial study, the inner surface of the coronal cavity was checked for microbial leakage. Sterile sharp spoon excavator was used to take samples for determination of the level of contamination from spacer material to access cavity. The collected sample was centrifuged using centrifuge machine. The collected sample was sent for microbiological examination to check for microbial growth on blood agar plates. A $0.1 \mathrm{ml}$ of sample was taken and inoculated on blood agar plate. Plates were incubated at $37^{\circ} \mathrm{c}$ for $24-36$ hours, the number of bacterial colonies were counted using a pen and click-counter in terms of $\mathrm{cfu} / \mathrm{ml}$ of the inoculum. The results obtained were tabulated and subjected to appropriate statistical analysis using ANOVA test. 


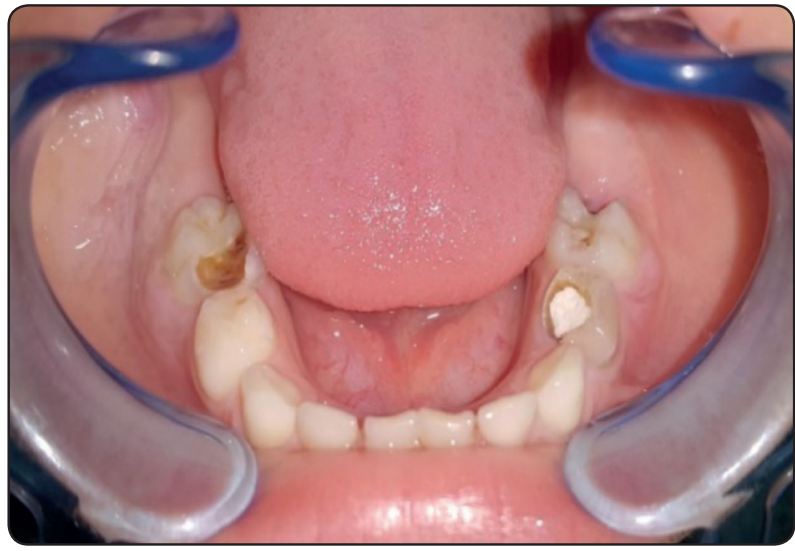

Fig. (1) Bilateral second primary molars indicated for pulpectomy

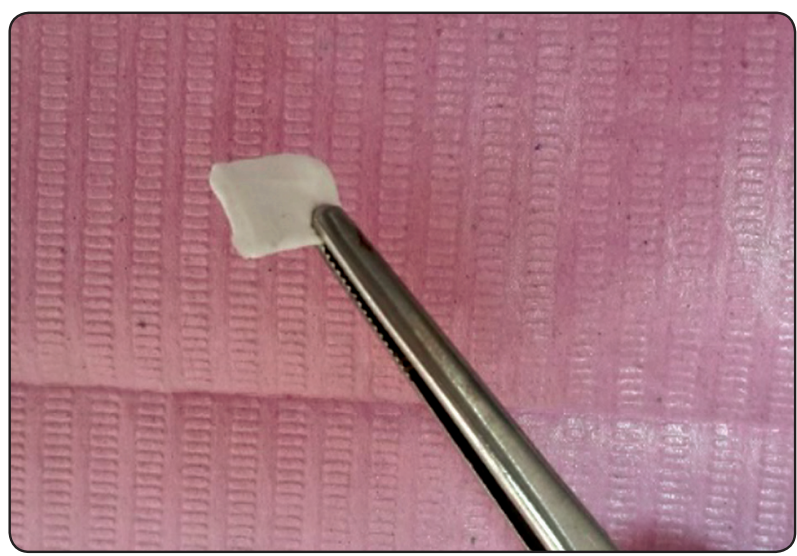

Fig. (3) Preparation of PTEF spacer

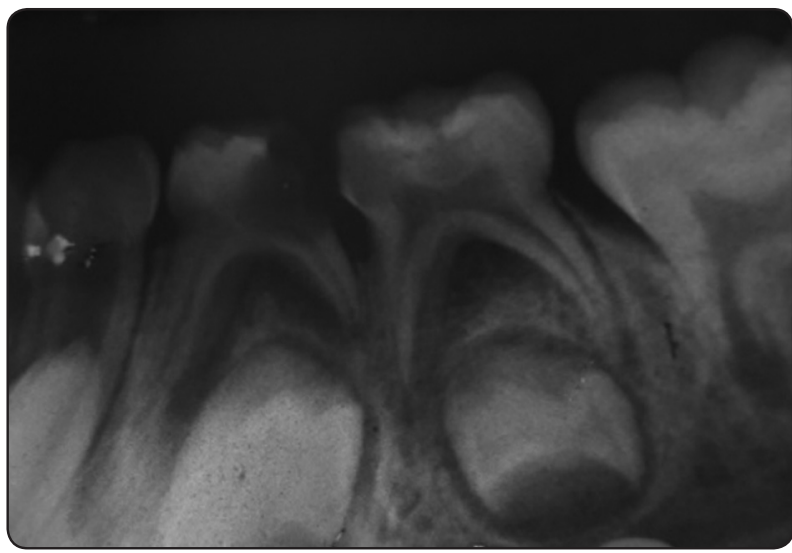

Fig. (5) Preoperative x-ray film of second primary molar

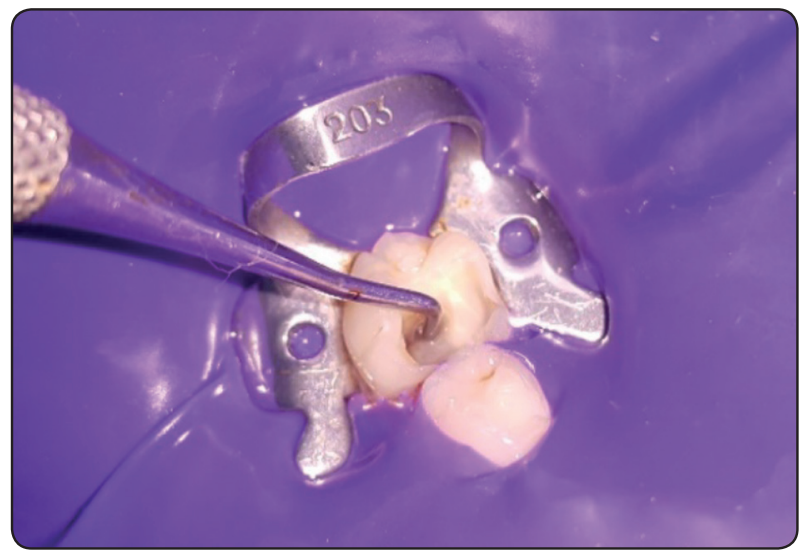

Fig. (2): Cavity after pulpectomy, taking fist sample

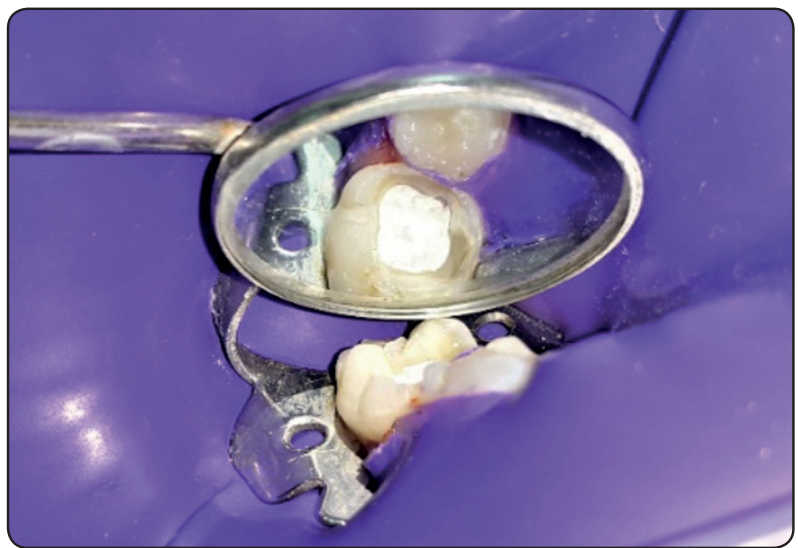

Fig. (4) Application of PTEF spacer in the cavity

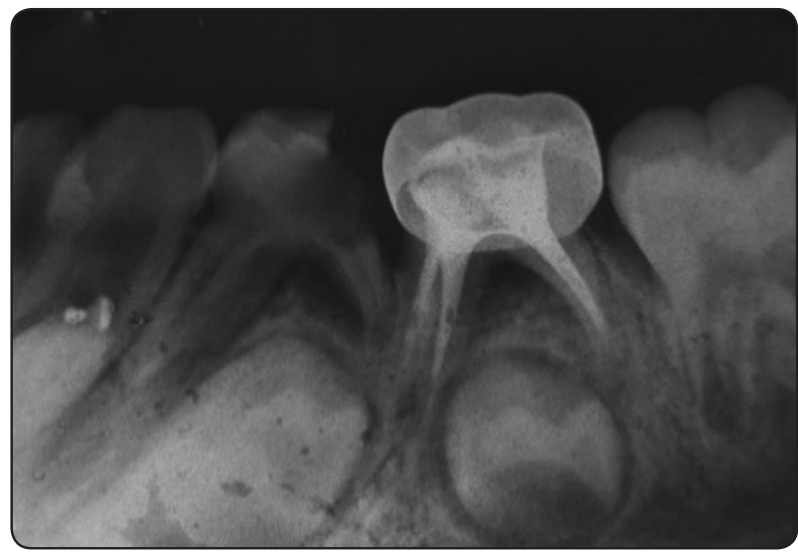

Fig. (6) Postopeartive periapical $x$-ray film of second primary molar 


\section{RESULTS}

Twenty children with forty primary molar teeth were included in this study aged from 6 to 8 years old with mean of 6.5. Females represent the majority of children (16) $80 \%$ of the tested groups. Most of the tested molars was the primary second molar (30) molars $75 \%$ while the first primary molars were 10 molar with percentage of $25 \%$ table (1)

TABLE (1) Distribution of participants and samples in the study

\begin{tabular}{|c|c|c|c|}
\hline Demographic variables & Group (I) & Group (II) & Total \\
\hline Age (years) & & & 6.5 \\
\hline Gender & & & \\
Male & 1 & 3 & 4 \\
Female & 9 & 7 & 16 \\
\hline First primary molars & 4 & 6 & 10 \\
Second primary molars & 19 & 11 & 30 \\
\hline
\end{tabular}

One child showed sever pain and swelling after two days of the obturation, so the case was excluded from the study and replaced by another one fig (7)

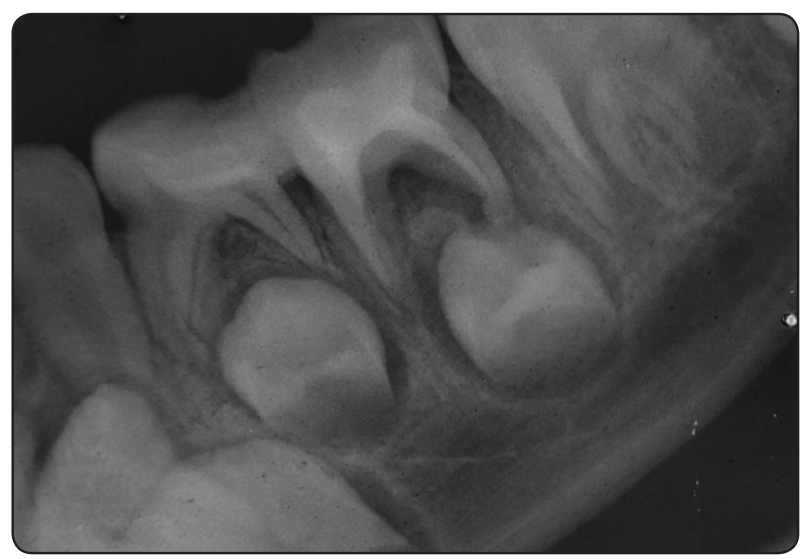

Fig. (7) Periapical x ray film for one of the excluded case from study

\section{Group I (Cotton spacer) bacterial incubation results}

The results of incubation of cotton samples in the blood agar media and counting the bacterial growth showed that there were no bacterial growth in the base line samples (Plate 1), but for the seven days samples, there was a significant increase in bacterial growth over the spacer material (Plate 2). In addition, there was a significant amount of microbial growth in the access cavity (Plate 3). Thus, microbial contamination was present on both spacer itself as well as in the access cavity. In addition, there was a significant difference in the mean colony count inside the group between that from the access cavity and spacer itself (Table II and Graph 1).

TABLE (2) microbial growth in group I base line and 7 days evaluation

\begin{tabular}{|c|c|c|c|}
\hline $\begin{array}{c}\text { Table 1 } \\
\text { Cotton spacer }\end{array}$ & Baseline & $\begin{array}{c}\text { Seven days } \\
\text { cotton culture }\end{array}$ & $\begin{array}{c}\text { Seven days } \\
\text { cavity } \\
\text { culture }\end{array}$ \\
\hline Range & $0-0$ & $12-47$ & $4-29$ \\
\hline Mean \pm SD & $0 \pm 0$ & $30.90 \pm 11.90$ & $15.60 \pm 7.99$ \\
\hline f. test & \multicolumn{3}{|c|}{96.695} \\
\hline p. value & \multicolumn{3}{|c|}{$0.001^{*}$} \\
\hline Baseline \& 7 days & $\begin{array}{c}\text { Baseline \& 7 } \\
\text { days cavity }\end{array}$ & \multicolumn{2}{|c|}{ cavity } \\
\hline $0.001 *$ & $0.001 *$ & \multicolumn{2}{|c|}{$0.001^{*}$} \\
\hline
\end{tabular}

$f:$ ANOVA test $; *$ Significant p value $<0.05$

\section{Group II (PTEF spacer) bacterial incubation re- sults}

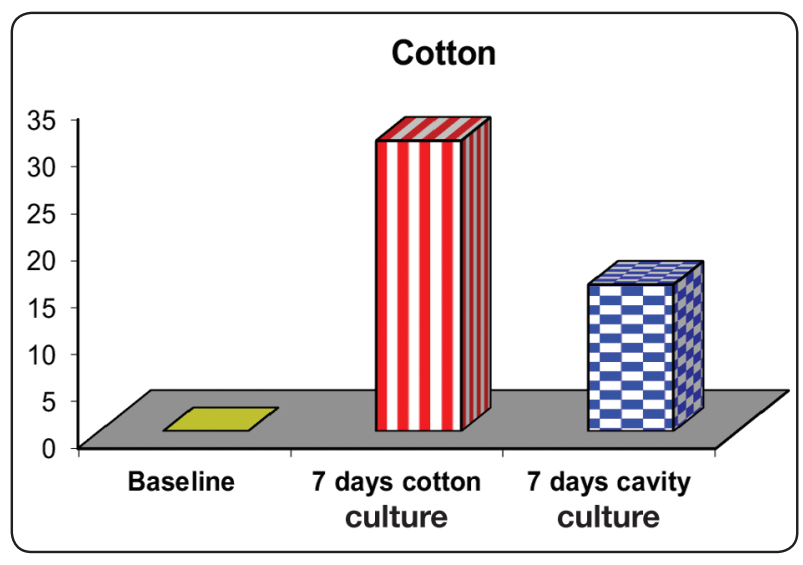

Graph I comparison of microbial growth in group I base line and seven days evaluation 


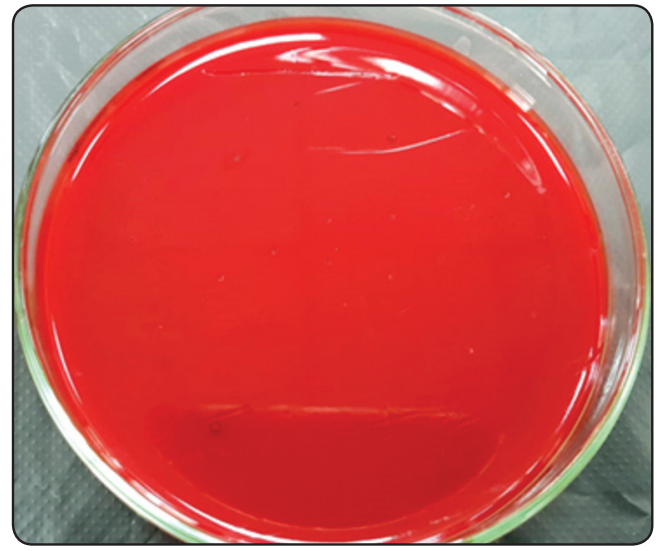

Plate (1): Blood agar plat demonstrated no microbial growth observed at baseline for group I

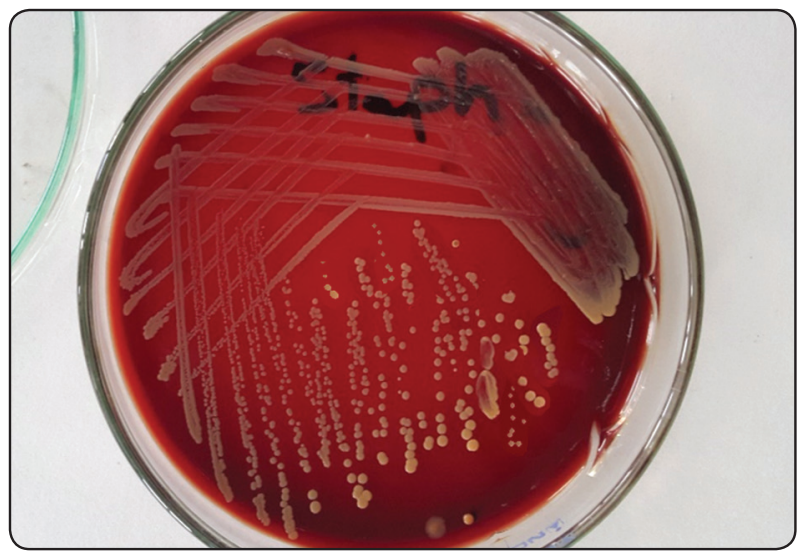

Plate (2): Cotton endodontic spacer material for group I: Over a period of seven days, there was a significant increase in microbial growth over the spacer material. Mostly Staphylococci.

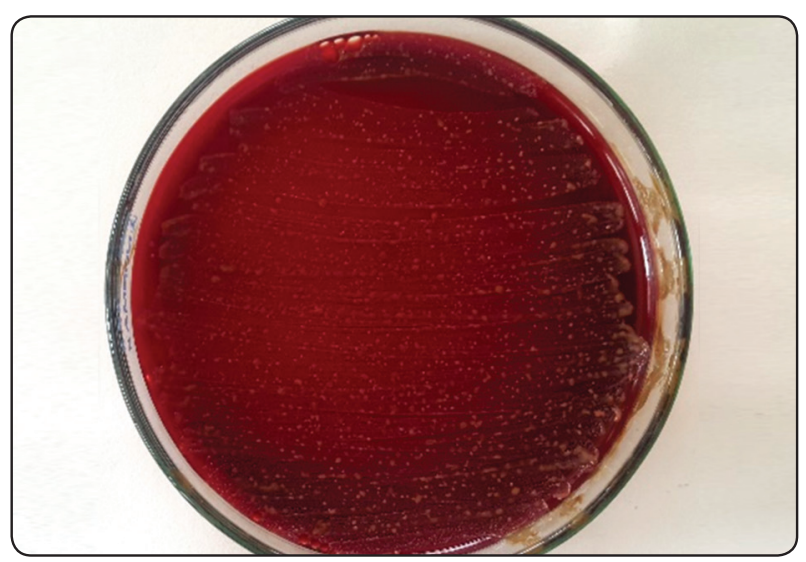

Plate (3): Access cavity material for group I, over a period of seven days; there was a significant increase in microbial growth over the spacer material. Mostly mixed growth gram +ve \& -ve organisms
The results of using PTEF as an endodontic spacer material demonstrated no microbial growth observed at baseline, but over a period of seven days, there was little increase in microbial growth over the spacer material (Plate 4). In addition, there was non-significant microbial growth in the access cavity (Plate 5). Thus, microbial contamination was minimal on both PTEF spacer as well as in the access cavity. (Table III and graph II).

TABLE (3) Comparison of microbial growth in group II (PTEF) base line and seven days evaluation

\begin{tabular}{|c|c|c|c|}
\hline PTEF & Baseline & $\begin{array}{c}\text { Seven days } \\
\text { PTEF } \\
\text { culture }\end{array}$ & $\begin{array}{c}\text { Seven days } \\
\text { cavity } \\
\text { culture }\end{array}$ \\
\hline Range & $0-0$ & $2-17$ & $0-7$ \\
\hline Mean \pm SD & $0 \pm 0$ & $9.05 \pm 3.90$ & $2.45 \pm 2.33$ \\
\hline f. test & \multicolumn{3}{|c|}{$\mathbf{6 3 . 7 3 7}$} \\
\hline p. value & \multicolumn{3}{|c|}{$0.001 *$} \\
\hline $\begin{array}{c}\text { Baseline \& seven } \\
\text { days }\end{array}$ & Baseline \& seven & Seven days \& seven \\
\hline $0.001 *$ & $0.001^{*}$ & \multicolumn{2}{c|}{$0.001 *$} \\
\hline
\end{tabular}

\section{$f:$ ANOVA test $; *$ Significant p value $<0.05$}

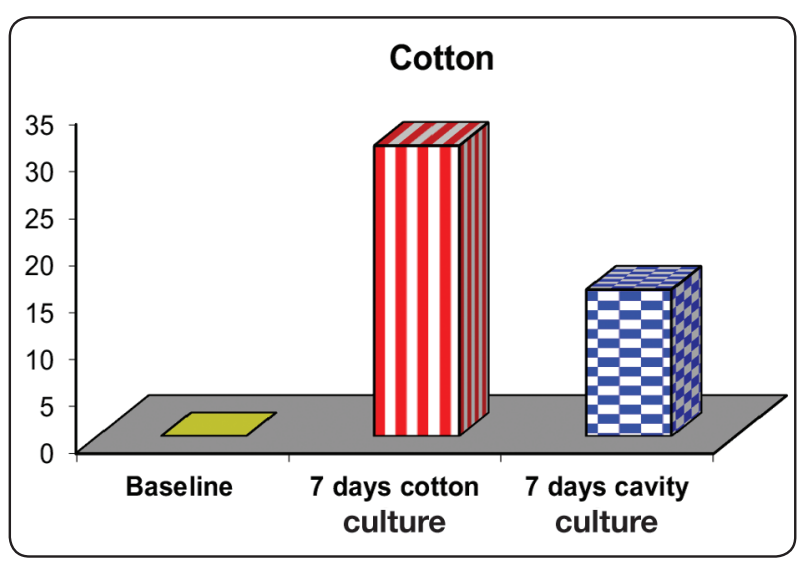

Graph II comparison of microbial growth in group II (PTEF) base line and 7 days evaluation 


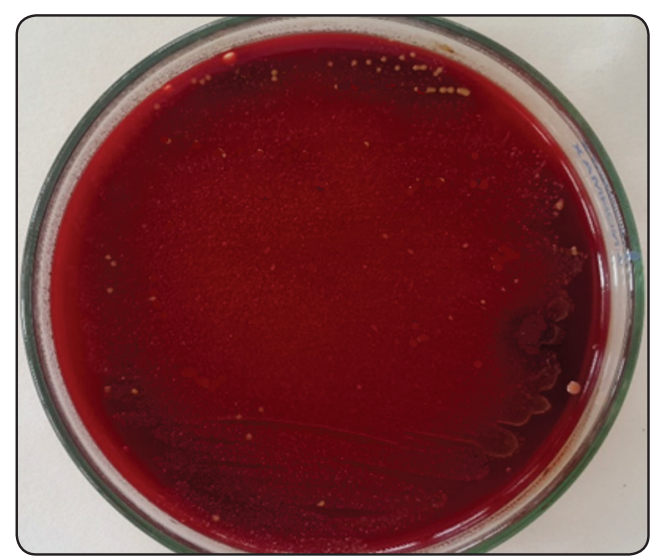

Plate (4): PTFE spacer material for group II: Over a period of seven days, there was a significant increase in microbial growth over the spacer material. Mostly G+ve organism

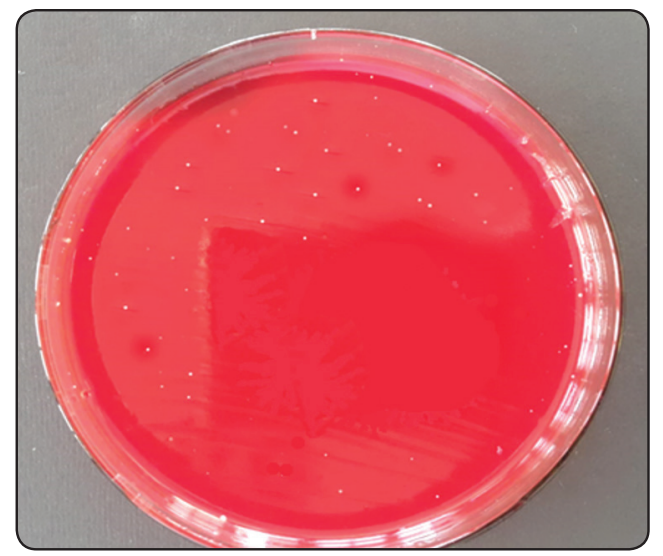

Plate (5): Access cavity material for group II PTFE: Over a period of seven days, there was a minimal microbial growth over the spacer material. Mostly G+ve and G-ve organism.

\section{Comparison between bacterial contamination on cotton and PTFE tape after seven days}

There was a significant difference in the colony count on cotton as compared to that of PTFE tape

PTFE tape showed less microbial contamination as compared to cotton as spacer material, also the colony count of PTFE tape showed less microbial leakage into the access cavity as compared to cotton as spacer material (Table IV and graph III).
TABLE (4) Comparison of microbial growth between group I and group II after 7 days in both the material and access cavity.

\begin{tabular}{|c|c|c|c|c|c|}
\hline & & Cotton & PTEF & T. test & P. value \\
\hline \multirow{2}{*}{ 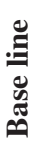 } & Range & $0-0$ & $0-0$ & \multirow[b]{2}{*}{ - } & \multirow[b]{2}{*}{ - } \\
\hline & $\begin{array}{c}\text { Mean } \\
\pm \text { SD }\end{array}$ & $0 \pm 0$ & $0 \pm 0$ & & \\
\hline \multirow{2}{*}{ 茓 } & Range & $12-47$ & $2-17$ & \multirow[b]{2}{*}{7.802} & \multirow[b]{2}{*}{$0.001 *$} \\
\hline & $\begin{array}{l}\text { Mean } \\
\pm \text { SD }\end{array}$ & $30.90 \pm 11.90$ & $9.05 \pm 3.90$ & & \\
\hline \multirow{2}{*}{ 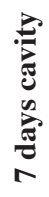 } & Range & $4-29$ & $0-7$ & \multirow[b]{2}{*}{7.067} & \multirow[b]{2}{*}{$0.001 *$} \\
\hline & $\begin{array}{c}\text { Mean } \\
\pm \text { SD }\end{array}$ & $15.60 \pm 7.99$ & $2.45 \pm 2.33$ & & \\
\hline
\end{tabular}

T: Independent sample T test $*$ Significant p value $<0.05$

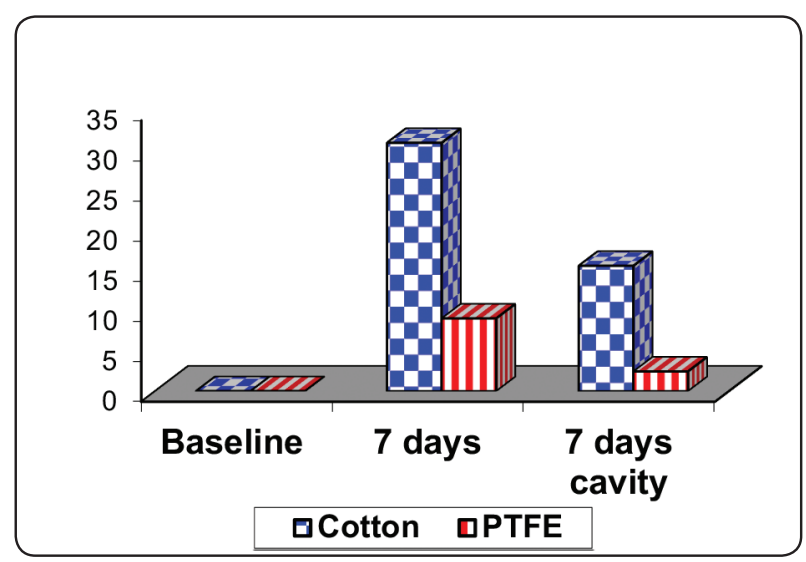

Graph (III) comparison of microbial growth between group I and group II after seven days in both the material and access cavity

\section{DISCUSSION}

The main target of pediatric dentist is to maintain the primary teeth in a non-pathologic and healthy condition for space maintenance, proper mastication, esthetics, phonetics and prevention of bad habits. Pulpectomy is a complete removal of necrotic pulp of primary teeth followed by obturation with inert resorbable material so as to maintain the tooth in the dental arch and at the same time allow normal physiological shedding of the primary teeth. 
Pulpectomy procedures for primary molars could be performed in one or multiple visits, when it is performed in multiple visits, temporary restorations are used to seal the access cavity preventing bacterial infection in between the visits. Beneath the temporary restorations, spacers were placed to make it is easy to remove the temporary restorative material, and also it acts as barrier between the restorative materials and root canals preventing it from coming inside the canals and also to facilitate relocation of the pulp chamber and the root canals ${ }^{(17)}$.

In this study we performed the multiple visits procedure using the spacer under the temporary restoration between visits to allow us to study the effectiveness of the new spacer material.

The selected age for this study was from 6 to 8 years to be sure that we have enough root length for successful pulpectomy procedures and also decrease the infection factor from the apical area, contralateral molars was selected for each child to have fixed oral and physiological factors.

Cavit was used in this study because it is routinely used by endodontists (28) and because previous in vitro and in vivo studies have shown it was performed the best results over the other temporary restorative materials ${ }^{(25),(29) \text {. }}$

Cotton is the most widely used spacer material all over the world under the provisional restorations in between the dental visits ${ }^{(26) .}$

Many clinicians use cotton pellets for application the medicaments into the restorative cavities. The removal of the cotton fibers considered one of the difficult steps because they frequently get attached to the cavity walls, potentially compromising the integrity of the definitive restoration. If a permanent restoration is not provided relatively soon after endodontic therapy, forces of mastication may cause wear of the surface of the temporary restoration, thereby reducing its thickness to below the critical $3.5 \mathrm{~mm}$ thickness. This may result in the exposure of the cotton fibers to the oral environment, which could lead to the starting of microbial contamination from the oral cavity and loss of the required proper seal ${ }^{(17)}$.

Many other materials were tried by practitioners as in between visits spacer to overcome the leakage tendency which suspected with cotton, like fibers, foam pellets and Polytetrafluoroethylene (PTFE) ${ }^{(17,23)}$.

PTFE tape, has many uses but its mainly used as plumber's tape, it has excellent physical properties supporting its effectiveness when used as spacer, for example, it is an inorganic, non-fibrous, ribbon like material. Also, it is inert, autoclavable, available and very economic.

Stean has reported several uses of PTFE tape in dentistry. He summarized its dental uses into, mechanical barrier applications, surgical uses and fit checker ${ }^{(27)}$.

This study carried out to compare the sealing ability of the traditionally used cotton fibers and the PTFE tapes underneath the temporary restoration in between visits during pulpectomy of primary molars.

PTFE tape when subjected to compression, it will forms a firm platform which could support the overlying restoration and reduce microbial leakage.

This study conducted clinically in forty primary molars teeth indicated for pulpectomy in a contralateral sides of twenty patients to assure standardization of the study.

Cavit was used in this study because it is routinely used by endodontists (28) and because previous studies have shown it performed better results than any other temporary material ${ }^{(25,29)}$.

The 7-day time point was selected based on previous in vitro and in vivo studies $(25,30)$.

The procedures followed in this study were similar to those of other studies that have evaluated leakage of Cavit restorations (25), (31). 
The length of PTFE tape was uniform to approximately $6 \mathrm{~cm}$ and the cotton used was also standardized for each sample. After placement of spacer, we used periodontal probe to measure the depth of the cavity to make sure that it will accommodate the required $3.5 \mathrm{~mm}$ thickness of the temporary restorative material as recommended by the other studies protocol $^{(32)}$.

Irrigation and cleaning of the cavity before taking the first sample was done only by normal saline to have the same environment like the regular root canal treatment procedures unlike other technique tried by Attiguppe et al $2018^{(7)}$ who used disinfectant(sodium hypochlorite) before taking the first sample, with the same result as our study which is no microbial growth in the first sample.

There was decrease of the number of bacterial growth underneath the temporary restoration isolated by PTFE than the one with cotton spacer this results coordinated with study performed by Attiguppe et al $2018^{(7)}$.

Attiguppe et al $2018^{(7)}$ concluded that there was a significant increase in the bacterial growth count after seven days in cotton group in contrary to our results in which there is increase in the bacterial count of the cotton group but with non-significant difference. The access cavities were also positive for microbial leakage in the cotton group which showed positive growth. In PTFE group only two samples showed microbial contamination and out of two only one sample showed access cavity contamination.

Efficacy of cotton and PTFE spacer was evaluated extra orally by Paranjpe et al $2012^{\left({ }^{(17)}\right.} .26$ extracted molars was used for their study, also the regular temporary restorative material(Cavit) used overlying the tested spacers After incubation for seven days in a culture of Streptococcus gordonii or in liquid media alone, bacterial contamination then tested for both the spacer material and the access cavity . Results revealed that only one tooth of the PTFE group was positive for S gordonii growth while nine of the ten teeth for the cotton group were positive both in the tested material and access cavity. And they reach to dramatically conclusion that even under complete isolation conditions, cotton spacers could allow for bacterial contamination into the access cavities. They thought that the cotton fibers may act as a reservoir for bacterial contamination of the access cavities and root canal space. In contrast, PTFE tape is an inert non fibrous and did not provide any way for bacterial contamination.

The good sealing result of PTFE may be attributed to easy handling and the no fibrous nature of the material and this also what thought by Attiguppe et al $2018^{(7)}$.

Our in vivo study is one of the pioneer studies about using PTFE as a spacer in pulpectomy of primary molars and we thought that it will be very beneficially for pediatric dentistry regarding higher success rate of two visits technique.

In vitro microbiological study by Aditya Shetty et al $2019^{(23)}$ consisted of three groups of spacers, Cotton, Cellulose sponge and PTFE tape. They compare it alone or with disinfectants (calcium hydroxide medicament and immersed in iodoform based medicament). The results showed very good seal with PTFE group compared to the other groups when used alone but not when used with medicaments.

The results of our study with other in vitro studies may make base for new era for two visits pulp therapy treatment techniques.

\section{CONCLUSION}

According to our study there was microbial contamination underneath the temporary restoration used between root canal visits of primary molars.

The microbial contamination with the PTFE group was too much less than that with cotton group, it needs more study to reveal the main cause of this contamination which may be related to the technique or the provisional material itself. 
PTFE tape is a simple, readily available and cost effective material that proved to be a successful when used as a spacer during two visits primary teeth endodontic treatment techniques. Further study needed to demonstrate the role of the cotton fibers in contamination of the cavity underneath the temporary restorative materials.

\section{REFERENCES}

1. American Academy of Pediatric Dentistry. Guideline on pulp therapy for primary and young permanent teeth. Pediatr Dent; 26:115-119. 2004.

2. Dummett CO Jr, Kopel HM. Pediatric endodontics. In: Ingle JI, Bakland LK, editors. Endodontics. $5^{\text {th }}$ ed. Hamilton: BC Decker Inc.; 2002. p. 861-902.

3. Ounsi HF, Debaybo D, Salameh Z, Chebaro A, Bassam H. Endodontic considerations in pediatric dentistry: A clinical perspective. Int Dent South Afr 2009; 11:40-50.

4. Guideline on pulp therapy for primary and immature permanent teeth. American Academy of Pediatric Dentistry - Reference Manual V 36 / NO 6 14/15.

5. Sjogren U, Figdor D, Persson S, Sundqvist G. Influence of infection at the time of root filling on the outcome of endodontic treatment of teeth with apical periodontitis. Int Endod J; Sep; 30(5):297-306. 1997.

6. Abbott PV (1994) Factors associated with continuing pain in endodontics.AustralianDentalJournal39, 157^ 61 .

7. Attiguppe Ramasetty, Kratika Dixit and Raju OS. Microbiologic Evaluation of Cotton and Polytetrafluoroethylene (PTFE) Tape as Endodontic Spacer Materials in Primary Molars An in Vivo Study. The Journal of Clinical Pediatric Dentistry. Volume 42, Number 1/2018 21-26.

8. Hommez GM, Coppens CR, De Moor RJ. Periapical health related to the quality of coronal restorations and root fillings. Int Endod J 2002; 35(8):680-689.

9. Schwartz RS, Fransman R. Adhesive dentistry and endodontics: Materials, clinical strategies and procedures for restoration of access cavities: A review. J Endod 2005; 31(3):151-165.

10. Ricucci D, Siqueira JF, Jr. Recurrent apical periodontitis and late endodontic treatment failure related to coronal leakage: A case report. J Endod 2011; 37(8):1171-1175.
11. Cardoso AS, Silva NC, Silva JM, Herrera DR, Neves AA, Leal Silva EJ. Assessment of coronal leakage of a new temporary light-curing filling material in endodontically treated teeth. Indian J Dent Res 2014; 25(3):321-324.

12. Siqueira JF, Jr., Rocas IN, Alves FR, Campos LC. Periradicular status related to the quality of coronal restorations and root canal fillings in a brazilian population. Oral Surg Oral Med Oral Pathol Oral Radiol Endod 2005; 100(3):369-374.

13. Ray HA, Trope M. Periapical status of endodontically treated teeth in relation to the technical quality of the root filling and the coronal restoration. Int Endod J 1995; 28(1):12-18.

14. Moreno JO, Alves FR, Goncalves LS, Martinez AM, Rocas IN, Siqueira JF, Jr. Periradicular status and quality of root canal fillings and coronal restorations in an urban colombian population. J Endod 2013; 39(5):600-604.

15. Siqueira JF, Jr., Rocas IN, Favieri A, Abad EC, Castro AJ, Gahyva SM. Bacterial leakage in coronally unsealed root canals obturated with 3 different techniques. Oral Surg Oral Med Oral Pathol Oral Radiol Endod 2000; 90(5):647-650.

16. 16-Webber RT, Del Rio CE, Brady JM, Segall RO. Sealing quality of a temporary filling material. Oral Surg Oral Med Oral Pathol; 46:123-130. 1978.

17. Paranjpe A, Jain S, Alibhai KZ, Wadhwani CP, Darveau $\mathrm{RP}$, Johnson JD. In vitro microbiologic evaluation of PTFE and cotton as spacer materials. Quintessence Int; 43(8):703-07. 2012.

18. Parris L, Kapsimalis P, Cobe HH, Evans R. The effect of temperature change on the sealing properties of temporary filling materials. II. Oral Surg Oral Med Oral Pathol 1964; 17:771-778.

19. Naoum HJ, Chandler NP. Temporization for endodontics. Int Endod J; Dec; 35(12):964-78. 2002.

20. Vail MM, Steffel CL. Preference of temporary restorations and spacers: A survey of Diplomates of the American Board of Endodontists. J Endod 2006; 32:513-515.

21. Newcomb BE, Clark SJ, Eleazer PD. Degradation of the sealing properties of a zinc oxide-calcium sulfate-based temporary filling material by entrapped cotton fibers. J En$\operatorname{dod} 2001 ; 27: 789-790$.

22. Sjogren U, Figdor D, Spangberg L, Sundqvist G. The antimicrobial effect of calcium hydroxide as a short-term intracanal dressing. Int Endod J; May; 24:119-25. 1991. 
23. Aditya Shetty, Payal Garg ,Mithra N, Hegde and Shishir Shetty: Microbiological Evaluation of Polytetrafluoroethylene (Ptfe) Tape, Cellulose Sponge and Cotton as Spacer Materials Combined with Intracanal Medicament- An in Vitro StudyIndian Journal of Public Health Research \& Development, March 2019, Vol.10, No. 3 61-65

24. Ciftci A, Vardarli DA, Sonmez IS. Coronal microleakage of four endodontic temporary restorative materials: An in vitro study. Oral Surg Oral Med Oral Pathol Oral Radiol Endod 2009; 108:e6 7-e70.

25. Beach CW, Calhoun JC, Bramwell JD, Hutter JW, Miller GA. Clinical evaluation of bacterial leakage of endodontic temporary filling materials. J Endod 1996; 22:459-462.

26. Vail MM, Steffel CL. Preference of temporary restorations and spacers: A survey of Diplomates of the American Board of Endodontists. J Endod 2006; 32:513-515.

27. Stean H. PTFE tape: A versatile material in restorative dentistry. Dent Update; 20:146-48. 1993.
28. Hartwell GR, Loucks CA, Reavley BA. Bacterial leakage of provisional restorative materials used in endodontics. Quintessence Int 2010; 41:335-339.

29. 29-Webber RT, del Rio CE, Brady JM, Segall RO. Sealing quality of a temporary filling material. Oral Surg Oral Med Oral Pathol 1978; 46:123-130.

30. 30- Trope M, Delano EO, Orstavik D. Endodontic treatment of teeth with apical periodontitis: Single vs. multivisit treatment. J Endod 1999; 25:345-350.

31. Ciftci A, Vardarli DA, Sonmez IS. Coronal microleakage of four endodontic temporary restorative materials: An in vitro study. Oral Surg Oral Med Oral Pathol Oral Radiol Endod; 108(4):67-70. 2009.

32. Odabas ME, Tulunoglu O, Ozalp SO, Bodur H. Microleakage of different temporary filling materials in primary teeth. J Clin Pediat Dent; Winter;34(2):157-60. 2009. 